\title{
OBSERVATIONS ON THE EXTENDED USE OF THE MARCHI METHOD
}

\author{
BY \\ MARION C. SMITH \\ From the National Hospital for Nervous Diseases, Queen Square, London
}

It has previously been reported (Smith, 1951) that the Marchi method applied to human material is useful in demonstrating degeneration in nerve fibres even when the interval between the occurrence of the lesion and death exceeded one year. Also (Smith, Strich, and Sharp, 1956) the Marchi method can be applied when the material has been stored in formol-saline for as long as eight years. This latter observation is contrary to traditional opinion ; it is important because it points to the possible use of the Marchi method on material that would previously have been considered totally unsuitable for this technique. The purpose of this paper is to report an investigation of the effects of storage in formol-saline on degenerating nerve fibres in human material, and to draw attention to the relation between the stage of degeneration and the kind of Marchi staining obtained.

The spinal cords from 18 patients were examined. Each patient had had a cordotomy at a known interval before death. In every case Marchi preparations were made after 10 to 14 days' fixation. During the next 15 to 72 months spare blocks of material were stored in $10 \%$ or $20 \%$ formol-saline. Slices were then cut from the blocks and a second series of Marchi preparations was made. The most satisfactory of these later preparations were found to be uncleared frozen sections prepared by the Swank-Davenport-Glees technique (Glees, 1943) but mounted directly after cutting in glycerine jelly (Smith and others, 1956) ; frozen sections cleared in cedar-wood oil and mounted in Canada balsam, and celloidin sections, some counterstained, were also prepared.

\section{Relationship between Period of Survival and Period of Fixation}

In every case the sections prepared after the short period of fixation contained black-staining, Marchipositive material. In the sections prepared after prolonged storage in formalin, on the other hand, black-stained, Marchi-positive material was present in some of the cases only, and there proved to be a correlation between the period of survival after a lesion and the occurrence of Marchi-positive material. After prolonged fixation it became evident that when the period of survival between the lesion and death was 75 days or less, Marchi-positive material was absent or present in only negligible amounts. When, however, the period of survival exceeded 75 days Marchi-positive staining material was present in every case. The amount of the Marchi-positive material in these cases varied in direct relation to the length of the period of survival after the lesion: thus in the cases with survival periods of 81 to 112 days there was much less material than in the cases with longer survival periods. The most vivid picture was obtained in the cases with survival periods of 390 and 466 days (Cases 55 and 56). This correlation between the length of the period of survival and the absence or presence of Marchi-positive material in sections prepared after prolonged storage in formol-saline is summarized in the Table.

Photographs from representative cases are shown on Page 69. In each case, photographs are given from the series of sections prepared after 14 days'

TABLE

FROZEN MARCHI PREPARATIONS MADE AFTER PROLONGED STORAGE OF TISSUE IN FORMOL-SALINE

\begin{tabular}{r|c|c|c}
\hline $\begin{array}{c}\text { Case } \\
\text { Number }\end{array}$ & $\begin{array}{c}\text { Period of Survival } \\
\text { after Cordotomy } \\
\text { (days) }\end{array}$ & $\begin{array}{c}\text { Period in } \\
\text { Formol-Saline } \\
\text { (months) }\end{array}$ & $\begin{array}{c}\text { Black-staining } \\
\text { Marchi-positive } \\
\text { Material }\end{array}$ \\
\hline 31 & 23 & 37 & $(+)$ \\
7 & 24 & 63 & - \\
33 & 39 & 35 & - \\
34 & 53 & 33 & - \\
2 & 75 & 66 & - \\
37 & 81 & 15 & - \\
38 & 87 & 24 & - \\
47 & 101 & 31 & - \\
32 & 101 & 60 & - \\
20 & (after left operation) & 64 & - \\
35 & 110 & 72 & - \\
10 & 112 & 65 & - \\
22 & 141 & 70 & - \\
21 & 154 & 31 & - \\
32 & 159 & 66 & + \\
35 & 172 & 42 & - \\
11 & (after right operation) & 23 & - \\
55 & 205 & 20 & - \\
56 & 239 & & \\
\hline
\end{tabular}


fixation (A) and from the series prepared after prolonged periods of fixation (B). The two series have been taken from the same region of the cord so that comparison of the areas of degeneration in the two sections in each case is justified.

In Case 34, Fig. 1 (period of survival, 53 days), the section prepared after 14 days' fixation (A) contains numerous black-stained bodies, indicating degeneration of nerve fibres, but in the section prepared after 33 months' fixation (B) there is no black staining, and therefore, in Marchi preparations, no evidence of any degeneration of nerve fibres.

In Case 37, Fig. 2 (period of survival, 81 days), there is abundant black-stained material in the section prepared after 14 days' fixation (A). In the section prepared after 15 months' fixation (B), there is a little black-stained material in the region of the most intense staining in the section prepared after short fixation.

In Case 47, Fig. 3 (period of survival, 101 days), black-stained material is present both in the section after 14 days' fixation (A) and that after 22 months' fixation (B), but it is rather more intensely stained in the section prepared after 14 days' fixation.

In Case 55, Fig. 4 (period of survival, 390 days), abundant black-stained material is present in the sections, both after 14 days' fixation (A) and after 43 months' fixation (B). The intensity of the staining is about the same in the two sections.

In the other cases in Table 1 , the findings were similar to those shown here. Within the limits of the material examined, the longer the period of survival after the lesion, the more vivid is the black zone of degeneration in the sections prepared after prolonged fixation.

When a lesion occurs three to 10 weeks before death, the period of fixation in formol-saline must be short for the satisfactory demonstration of degenerating myelinated fibres by the Marchi method; when the lesion occurs 10 weeks to 15 months before death, the period of fixation in formol-saline can be short or long. Most of the Marchi-positive material is not demonstrable in tissue kept in formol-saline for 30 months (or possibly less) when the lesion occurred three to 10 weeks before death. When the time between the lesion and death is an intermediate period, about 10 to 16 weeks, the degeneration is more clearly shown up when the period of fixation in formolsaline is short. When the time between the lesion and death is six months or longer the degeneration is as clearly shown up after long as after short periods of fixation.

\section{Extracellular and Intracellular Marchi-positive} Material

These conclusions lead one to ask the question : What changes occur in the black-staining material during the various periods of survival, so that it reacts differently to short and to long periods of storage in formol-saline? A histological answer to this question will now be given. When the black-a. stained material is examined under higher magnifi? cation, certain differences are seen between the material from cases with short survival periods and that from cases with long survival periods.

On pages 70 and 71 are photographs which are magnified views from those on page 69 (the areamagnified is shown in the small diagrams). On pages 70 and 71 , as on page 69 , the (A) figures arew from the sections prepared after 14 days' fixation $\overrightarrow{0}$ and the (B) figures are from those prepared afterprolonged periods of fixation. In addition to thew figures derived from those on page 69 , there are two? extra figures: Fig. $5 \mathrm{~A}^{\prime}$ is a photograph of a longitudinal section from Case 34, prepared after 14 days?. fixation, and Fig. $8 \mathrm{~B}^{\prime}$ is a frozen section frof Case 55 prepared after 43 months' fixation afdos dehydrated and cleared before mounting.

In Case 34, shown in Fig. 5A, $A^{\prime}$ and $B$, the period of survival was 53 days.

In Figs. 5A and $\mathrm{A}^{\prime}$ the black-stained materan consists of swollen and disrupting myelin shea出sbreaking up into globules. No scavenger cells cir taining black globules can be seen. This is classical picture of degenerating nerve fibres as demonstrated by the Marchi method. In Fig. 5B, which is a comparable section made after prolongedo fixation in formol-saline, there is almost no blackő staining to be seen. All the other cases in the group surviving 21 to 75 days present a similar picture; very $\overrightarrow{\vec{F}}$ little black staining, or none at all, was found after 3 prolonged fixation.

In Case 37, shown in Figs. 6A and $\mathrm{B}$, the periodo of survival was 81 days. In Fig. 6A there is more abundant degeneration than in Fig. 5A, but the pattern of numerous rounded bodies of differentosizes is comparable in the two cases. The bulk of . the black staining material appears to be extra-i cellular. In Fig. 6B there are some black-stained3 bodies, but these are very few compared with theo number stained in the section prepared after 14 days' fixation. There are also areas of indefinite, greyish, rather "granular" staining in the background.

In Case 47, shown in Figs. 7A and B, the period of 0 survival was 101 days. There is abundant staining in the preparations after short periods of fixationo 

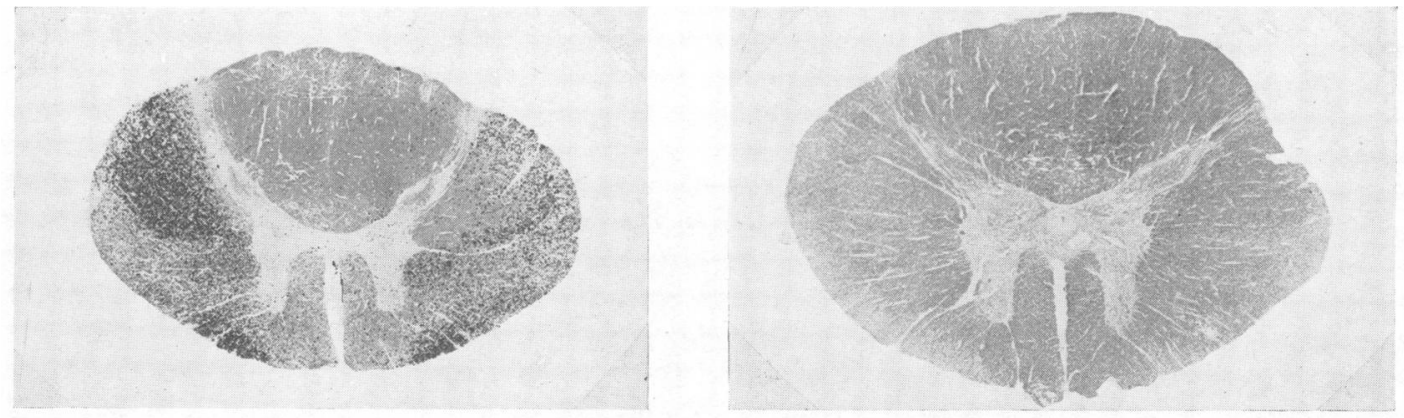

A

P.O.F. 14 days

P.O.F. 33 months

Fig. I.-Case 34 : P.O.S. 53 days
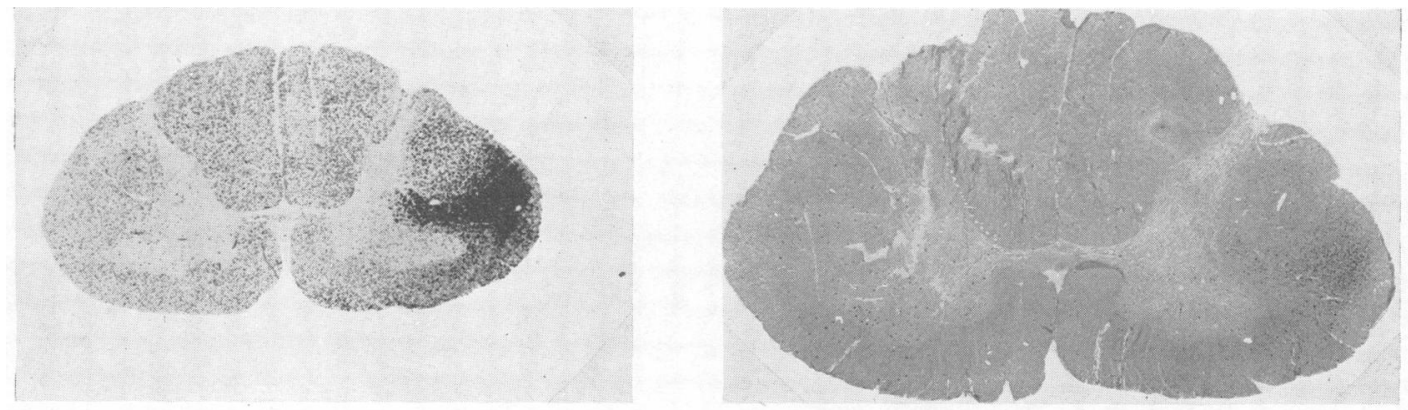

A

P.O.F. 14 days

P.O.F. 15 months

Fig. 2.-Case 37 : P.O.S. 81 days
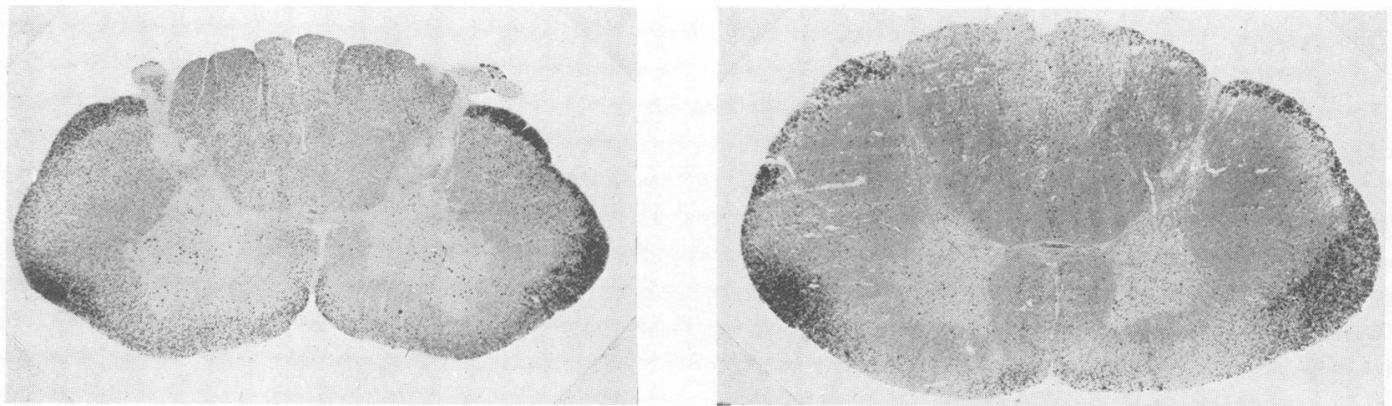

A

P.O.F. 14 days

P.O.F. 22 months

B

Fig. 3.-Case 47 : P.O.S. 101 days
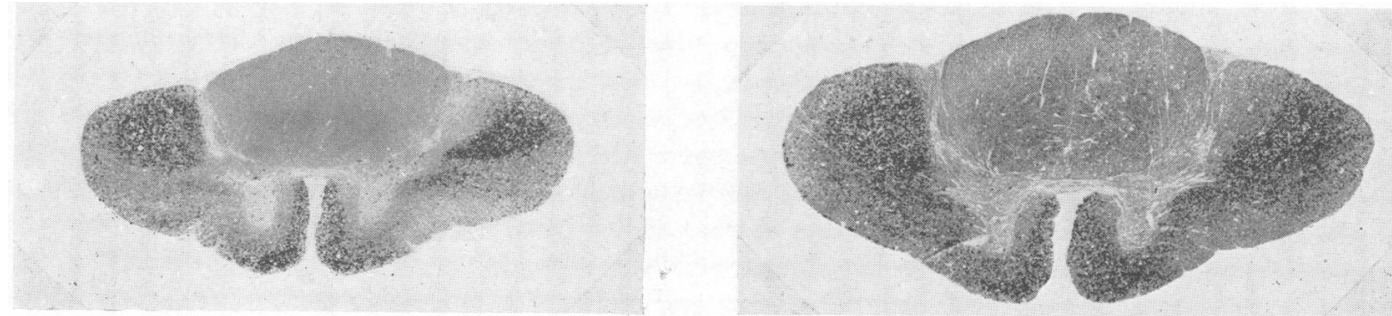

A

P.O.F. 14 days

P.O.F. 43 months

B

Fig. 4.-Case 55 : P.O.S. 390 days

P.O.S. $=$ period of survival ; P.O.F. $=$ period of fixation . 


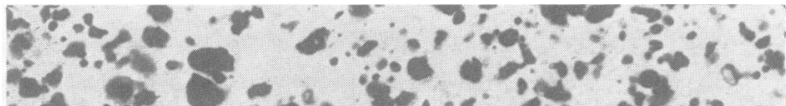

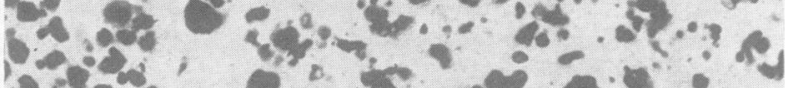

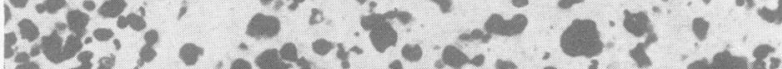
a $\because$ is

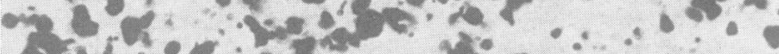

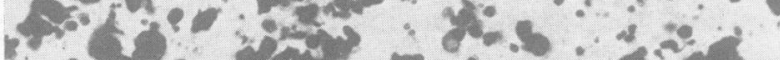

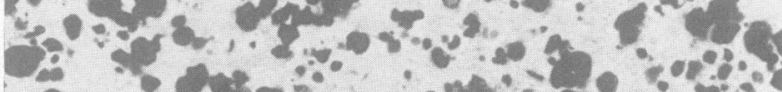
a

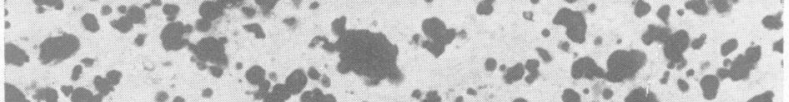

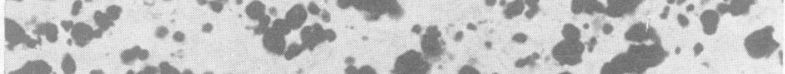

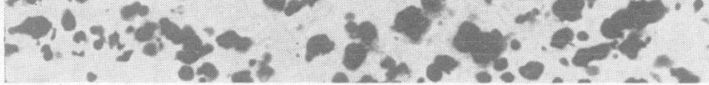

A
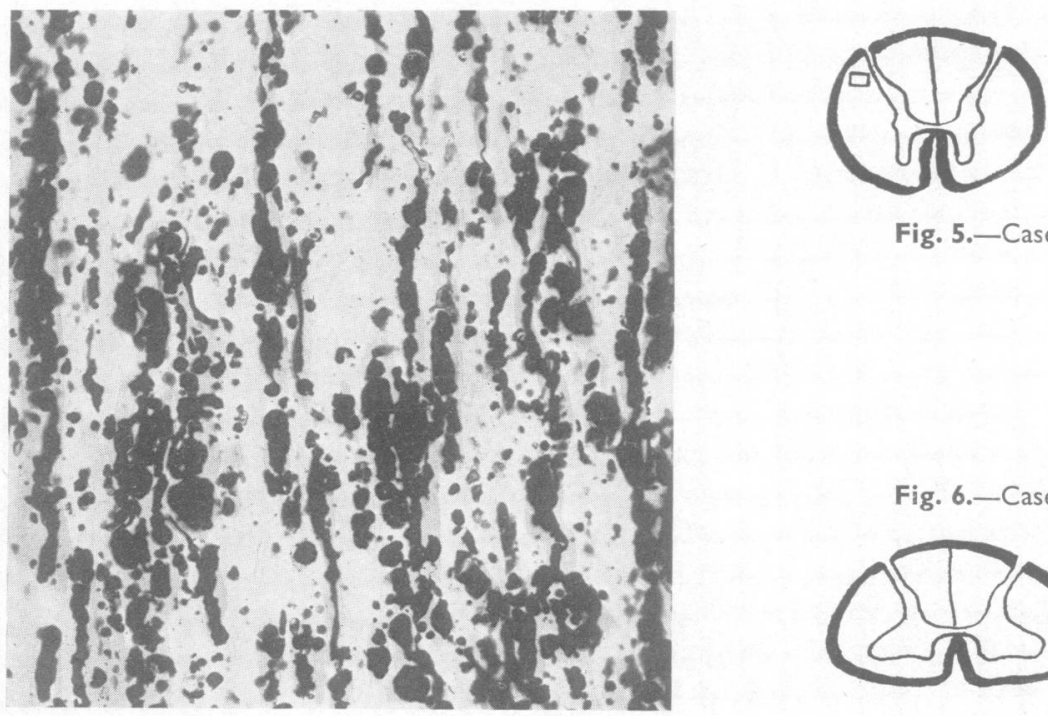

A : P.O.F. 14 days

$\mathbf{A}^{\prime}$ : P.O.F. 14 days

(longitudinal section:

Fig. 5.-Case 34 : P.O.S. 53 days

B : P.O.F. 33 months ợ
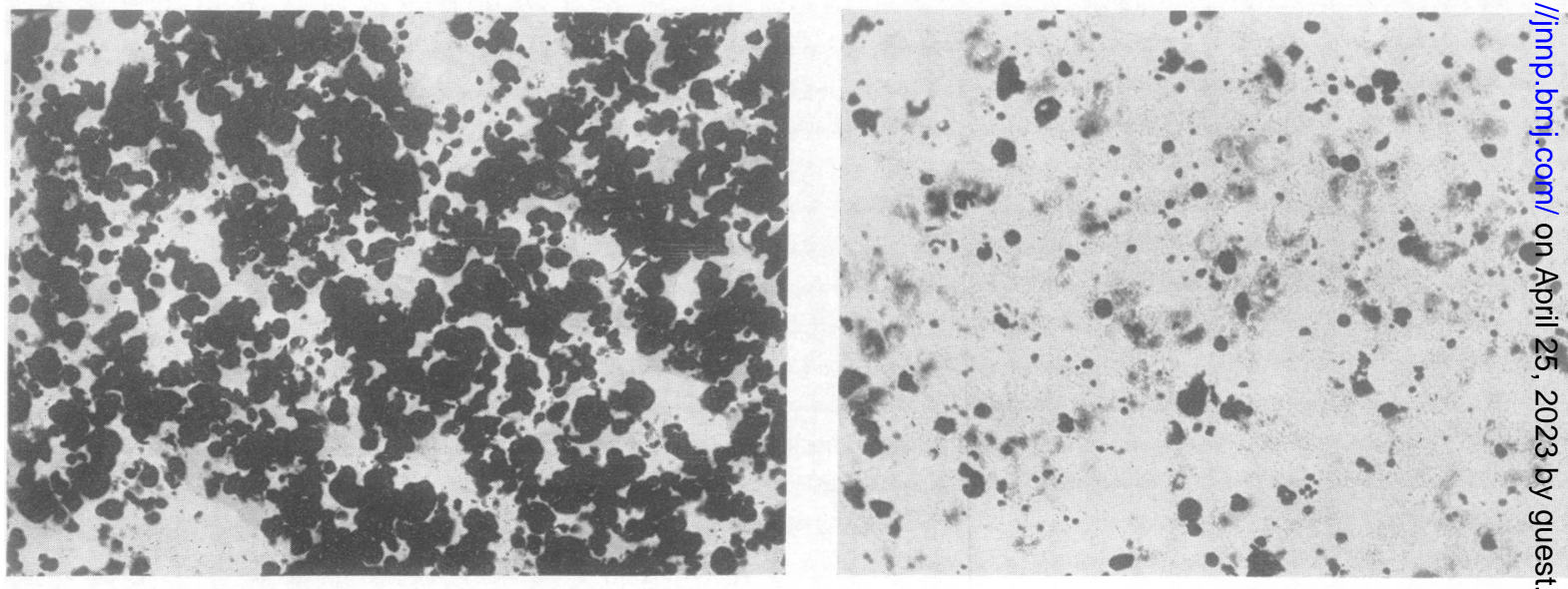


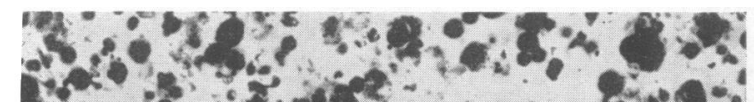

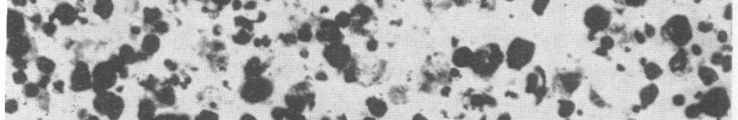

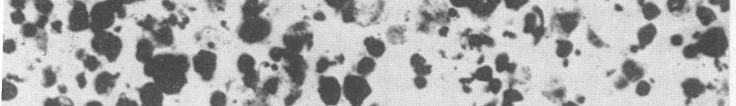

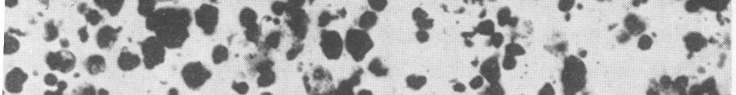
- 3

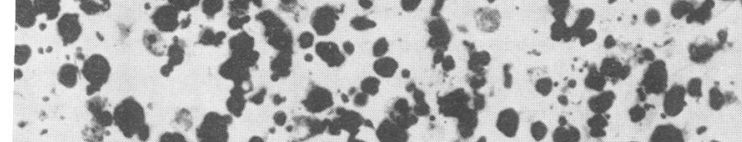

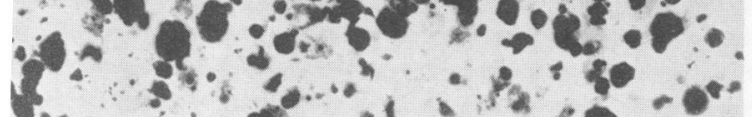

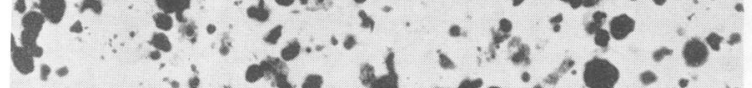
$\because-10$ of $\div$ ? ?

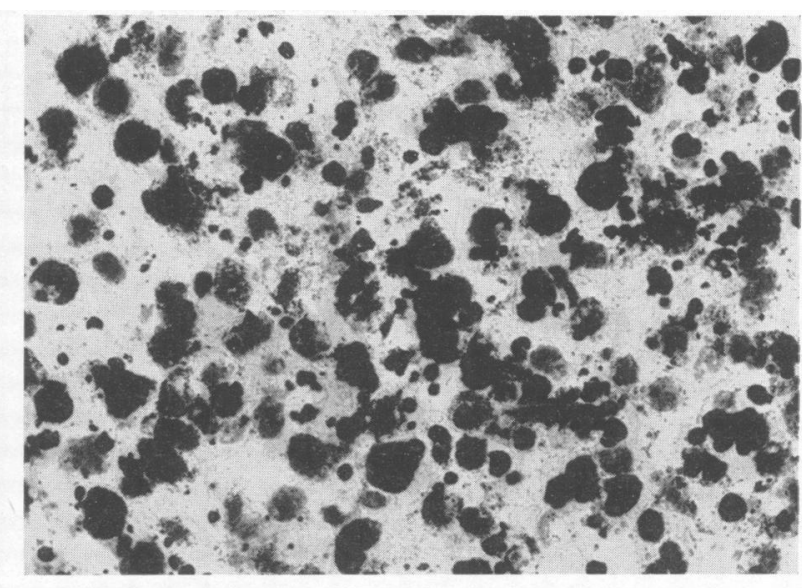

A : P.O.F. 14 days

B : P.O.F. 22 months

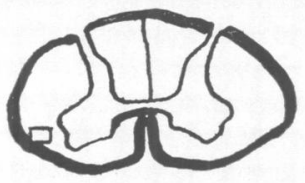

Fig. 7.-Case 47 : P.O.S. I0I days

Fig. 8.-Case 55 : P.O.S. 390 days

A : P.O.F. 14 days

B : P.O.F. 43 months

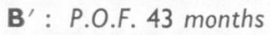

(cleared section)
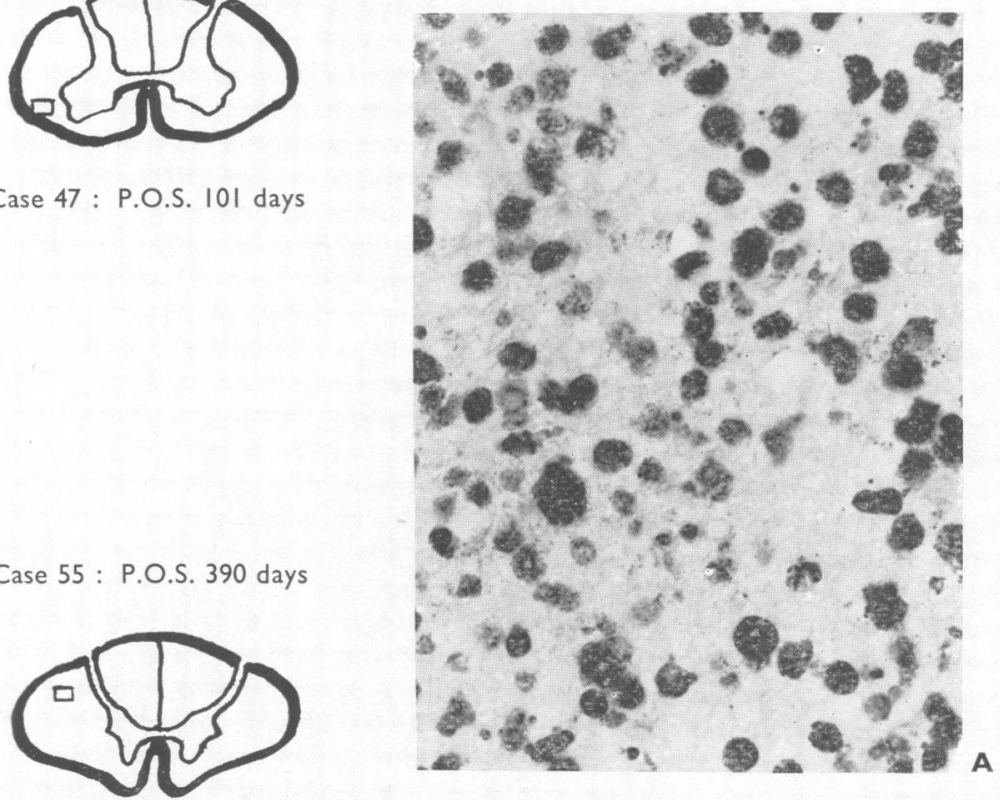

B

$\underset{\mathbb{D}}{\stackrel{C}{c}}$

으

$\stackrel{\mathbb{D}}{=}$

气

$\underbrace{0}_{0}$

高.

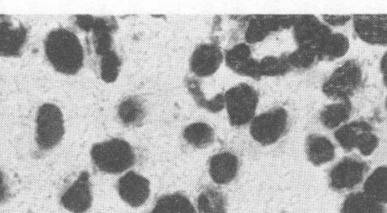

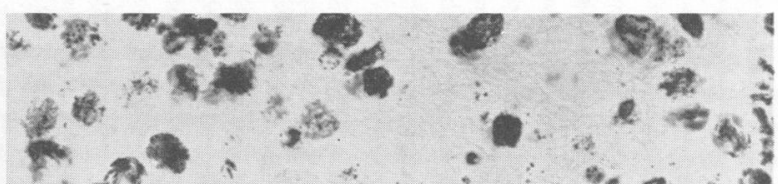

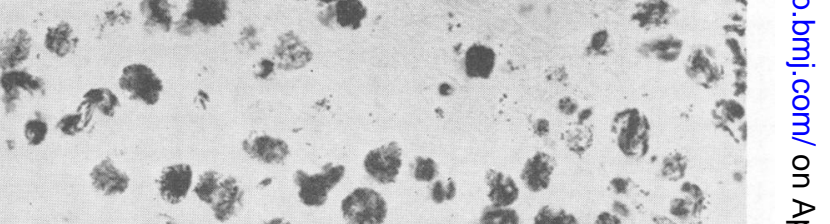

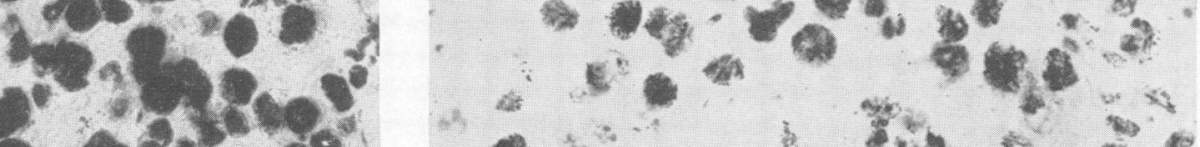

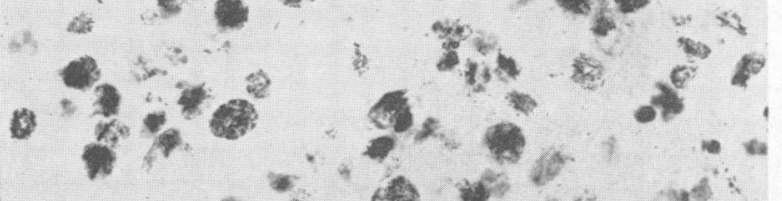
울 일ㅇ 
and also after prolonged fixation. In Fig. 7A there are numerous black bodies similar to those seen in Figs. 5A and 6A, and many of these do not appear to be contained in cells ; there are also numerous "groups" of greyish "globules" which do not seem to be lying free but to be contained within cells. In Fig. 7B there are large regular black bodies, some smaller black bodies, and many groups of small black or grey " globules." In some of these groups there is also a larger black body.

In Case 55, shown in Figs. 8A, B, and $\mathrm{B}^{\prime}$, the period of survival was 390 days. In Fig. 8A the appearance of the black-stained material is much more regular than in the other three cases. There are few, if any, extracellular black-staining bodies of varying size, as are present in the other cases. The black material appears to be entirely intracellular, and to be in small "globules". Some of the cells contain globules which stain a greyish colour, others are intensely black. In the uncleared section prepared after prolonged fixation, the black material is in the form of rather regular homogeneous looking, black masses; in the cleared preparation, $\mathbf{B}^{\prime}$, a picture comparable to $7 \mathrm{~A}$ is obtained. It is evident that the black and grey globules are contained in cells, which must be compound granular corpuscles. The homogeneous masses in the uncleared preparation, Fig. $8 \mathrm{~B}$, are obviously the same intracellular material seen in Fig. $8 \mathrm{~B}^{\prime}$. The preparations made after short and after prolonged periods of survival are more comparable in this case than in the cases with shorter survival periods. If Fig. $6 \mathrm{~B}$ is now reexamincd, it can be seen that the groups of greyish material resemble the intracellular material in Figs. $7 \mathrm{~B}$ and $8 \mathrm{~B}$ and $\mathrm{B}^{\prime}$. It seems likely that they are scavenger cells in this case also.

If all the (A) preparations are now re-examined, it can be seen that in the cases with periods of survival of 53 to 81 days, little, if any, of the black material is intracellular and that most of it lies free in the tissue. In the case surviving 101 days (Case 47) some of the material is intracellular and some is extracellular. In the case surviving $\mathbf{3 9 0}$ days (Case 55 ), all the material is intracellular.

If all the (B) series of preparations are examined, it can be seen that in the case surviving 53 days (Case 34) extremely little of the degenerated material has been stained. In the case surviving 81 days (Case 37), part of the extracellular material has stained. A little of the intracellular material has also stained, some of the globules being black and others grey. Fig. 7B and Figs. 8B and $\mathrm{B}^{\prime}$ are similar in that the black material in Fig. 7B is mainly, and in Figs. 8B and $B^{\prime}$ entirely, intracellular.
From these cases and the other cases in the series certain conclusions can be drawn.

It is apparent that the Marchi method stains black certain degeneration products of myelin, 只 whether the material is situated extracellularly 0 amongst the nerve fibres or intracellularly in $D$ scavenger cells. The appearance of the products of degenerating fibres is dependent not only on the length of the period of survival, but also on the length of the period of fixation. The amount of extracellular material which is stained black is $\overrightarrow{\bar{s}}$ greatest when the period of fixation is short. With $\overrightarrow{0}$ longer periods of fixation there is a gradual diminution in the amount of extracellular material demonstrable by the Marchi method, and eventually, after prolonged periods of fixation, no extracellular material at all can be demonstrated. It appears to ${ }^{\infty}$ be probable that the longer the period of survival, the $\vec{\circ}$ longer can the tissue be stored in formol-saline and some extracellular material still be demonstrated. $\stackrel{\omega}{\sigma}$ The exact lengths of time during which cases with $\overline{\overline{3}}$ periods of survival of less than 80 days can be? stored in formol-saline and extracellular material $\vec{\omega}$ still be stained has to be determined. With respegt in to the intracellular material, much of it is stainem however long the tissue has been preserved in formo? saline. Although to a markedly less extent than wiff the extracellular material, it appears to be likefy $\bar{z}$ that there is some correlation between the lengel of the period of survival and the amount of intris cellular material still demonstrable after prolonge्. fixation. The longer the period of survival, with certain limits, the longer can material be stored in formol-saline, and as much intracellular Marchipositive material as was stained after short periods of fixation still be demonstrated. But it must be stressed that prolonged periods of fixation do not $\stackrel{\mathbb{Q}}{\Omega}$ alter the positive Marchi picture to anything like $\overrightarrow{\overrightarrow{0}}$ the same extent when the material is in the intra- 3 cellular stage as when it is in the extracellular stage.

It is to be noted that the distribution of the degenerating fibres indicated by black staining in the Marchi preparations corresponds exactly to the distribution indicated by other staining methods, whether the period of survival was short or long. It can then be concluded that the black-staining material remains in approximately its original site, $₹$ even when the period of survival is more than a year. This applies to the intracellular material as $>$ well as to the material lying free between the fibre mesh.

This finding, that the material derived from degenerating myelin remains in situ for at least $15^{\mathrm{N}}$ months, is surprising; for it might have been thought that once the material had been taken up 
by scavenger cells it would have been removed from the site of degeneration and have entered the blood stream. As this intracellular black-staining material remains in situ, the Marchi method is still useful in tracing degeneration when the period between the lesion and death is such that these products of myelin degeneration, which stain black, are all takén up by scavenger cells.

The amount of intracellular black-staining material is of course related to the amount originally available from the myelinated nerve fibres; it follows that where a tract consists of thinly myelinated fibres the Marchi method applied after prolonged fixation will not give good staining, any more than it does in the early stages of fixation.

\section{Estimation of Period of Degeneration by the Marchi Method}

In all the cases described here, the lesion which caused degeneration in the nerve fibres was a surgical one, consisting of actual transection of the fibres ; the exact period of survival after the lesion was known. From these cases, the appearances of degeneration at the various intervals between the lesion and the death of the patient, as shown by the Marchi method, can be seen. Thus a series such as these cases with lesions made at known intervals between 21 and 466 days before death can be used as a standard of comparison for estimating when a lesion occurred, and also whether the degeneration seen in nerve fibres occurred all at one time or if it occurred in more than one stage. It is probable that a full comparison can justifiably be made between the series of cases reported here and other cases in which the lesion is traumatic. But whether the process of degeneration in other pathological conditions is similar enough to warrant a direct comparison remains to be investigated. In such cases, even if conclusions as to the duration of the condition cannot be drawn, at least it can be concluded whether the degeneration occurred at one time, or continuously, or in stages at dispersed intervals.

In traumatic cases, when there is abundant extracellular black-staining material, and very little intracellular material, it may be concluded that the degeneration of nerve fibres has been going on for less than $\mathbf{7 5}$ days. When there is a moderate amount of intracellular material, staining grey in many cells, and staining black in a few cells as well as in some extracellular material, it is probable that the degeneration of nerve fibres occurred three to four months before death. If in addition to the intracellular material there are very large amounts of extracellular material, then the degeneration may be assumed to be continuous, or to have been repeated during the three to four months preceding death. When there are numerous cells containing black-staining material, only a few cells containing grey-staining material, and no extracellular material, the degeneration of the fibres may be taken as having occurred about a year before death. The presence of many grey-staining scavenger cells and/ or free black-staining material would indicate more recent degeneration also.

Perhaps it should be added that this use of the Marchi method to estimate the duration of a lesion is not designed to replace other histological methods of doing this but to supplement them.

\section{Summary}

An investigation was undertaken of the application of the Marchi method to tissue stored for various periods in formol-saline. It was found that all the black-staining, Marchi-positive material resulting from degeneration of nerve fibres is at first extracellular and later intracellular. For approximately 10 weeks after a lesion the material is mainly extracellular, after this time an increasing amount of the material is intracellular, and by 12 months after the lesion the material is entirely intracellular. When it is in the extracellular state the material can be stained very clearly with the Marchi method after limited periods of fixation, but most of it does not stain when the material has been fixed for two and a half years or longer. When it is in the intracellular state much of it stains clearly after both limited and prolonged periods of fixation in formol-saline. In material in which the lesion occurred a year or longer before death, particularly vivid staining is obtained after prolonged fixation.

It is a pleasure to thank Dr. E. A. Carmichael for his help and encouragement in this investigation, Mrs. J. A. Mills and Mr. Peter Sharp for all the technical work involved, and Mr. J. A. Mills for his kindness in taking the photographs.

\section{REFERENCES}

Glees, P. (1943). Brain, 66, 229. Glees, P. (1943). Brain, 66, 229.
Smith, M. C. (1951). Journal of Neurology, Neurosurgery and
Psychiatry, 14, 222.

Strich, S. J., and Sharp, P. (1956). Ibid., 19, 62. 